\title{
Intraoperative Activation of Very Wide Range Cold Agglutinins during a Cardiac Bypass Case
}

\author{
Saurin J Shah, $M D^{1^{*}}$, Juan C Mora, $M D^{1}$, Shashank S Shettar, $M D^{2}$ and John P Pirris, $M D^{3}$ \\ ${ }^{1}$ Department of Anesthesiology, University of Florida College of Medicine, USA \\ ${ }^{2}$ Department of Anesthesiology, The University of Oklahoma Health Sciences Center, USA \\ ${ }^{3}$ Department of Surgery, University of Florida College of Medicine, USA
}

*Corresponding author: Saurin J Shah, MD, Department of Anesthesiology, University of Florida College of Medicine, Jacksonville, 655 West Eighth Street, Jacksonville, FL 32209, USA, Tel: (904)-244-4351

\begin{abstract}
Cold agglutinin disease is a rare form of autoimmune hemolytic anemia mediated mainly by IgM antibodies reacting to surface antigens of the red blood cells at low temperatures causing agglutination and posterior hemolysis on rewarming. Patients with this condition might never be symptomatic, but when they are exposed to hypothermia during cardiovascular procedures, these reactions can be triggered leading to serious organ damage. We describe/discuss a case of a patient with a wide range cold agglutinin disease that became active during coronary artery bypass grafting, the subsequent management and current approaches to this entity in the perioperative setting.
\end{abstract}

\section{Keywords}

Cold agglutinin, Thermal range, Hemolysis, Hypothermia

\section{Introduction}

Cold agglutinins (CAs) are autoantibodies that become active at temperatures below physiologic body temperature causing the red blood cells (RBC) to agglutinate leading to increased viscosity, complement fixation and subsequent hemolysis with potential for lethal complications of microvascular occlusion and organ failure [1]. Clinical severity is more closely related to the titer of the CAs and their thermal range. Although present in most individuals, CAs are rarely of clinical significance as they are usually benign, polyclonal and become active only on exposure to cold [2]. Cardiac surgical procedures requiring intraoperative hypothermia are unique in creating a con- ducive thermal milieu for these CAs to be unmasked and have clinically substantial implications. Whether and to what degree CAs achieve significant RBC agglutination, or frank hemolysis during systemic hypothermia is unknown [3]. We report on a patient who was found to have CAs with extremely wide thermal range unrecognized until intraoperative problems were encountered, complicated by acute hemiplegia postoperatively with complete neurological recovery. While not the first case reported, it is very rare to encounter patients with such a wide thermal activation range.

\section{Case Presentation}

A 71-years-old male with past medical history of hypertension, hyperlipidemia and heavy smoking presented with STEMI. Cardiac catheterization revealed total ostial right coronary occlusion and severe left anterior descending artery disease not amenable to medical management warranting surgical intervention. Patient was scheduled for a two vessel coronary artery bypass grafting (CABG). He underwent extensive preoperative workup which included echocardiogram, carotid ultrasound, pulmonary function testing and was monitored for acute coronary syndrome during his week-long stay in the cardiac ICU.

Arterial line was placed pre-induction. General endotracheal anesthesia was induced with fentanyl

Citation: Shah SJ, Mora JC, Shettar SS, Pirris JP (2020) Intraoperative Activation of Very Wide Range Cold Agglutinins during a Cardiac Bypass Case. Int J Anesthetic Anesthesiol 7:105. doi.org/10.23937/23774630/1410105

Accepted: April 20, 2020: Published: April 22, 2020

Copyright: (C) 2020 Shah SJ, et al. This is an open-access article distributed under the terms of the Creative Commons Attribution License, which permits unrestricted use, distribution, and reproduction in any medium, provided the original author and source are credited. 
$500 \mathrm{mg}$, propofol $50 \mathrm{mg}$ and rocuronium $60 \mathrm{mg}$ using standard ASA monitors along with cerebral oximetry, bispectral index (BIS) and transesophageal echocardiography. ACT-guided heparin was administered for anticoagulation prior to aortic cross clamping. Upon initiation of cardiopulmonary bypass, retrograde cold cardioplegia was used to arrest the heart and patient was cooled down to $33.8{ }^{\circ} \mathrm{C}$. During the coronary anastomoses, the cardioplegia line was observed to have high pressures and became partially occluded. An increased amount of sludging and small clots were also observed by the surgeon, which was highly uncommon in the presence of adequate anticoagulation as monitored by the ACT. A review of the patient's electronic medical record (EMR) revealed presence of a cold autoantibody that was not picked up preoperatively. The cold antibody had a wide thermal range from $4{ }^{\circ} \mathrm{C}$ to $37{ }^{\circ} \mathrm{C}$ with a high titter. The surgeon immediately administered copious amounts of warm cardioplegia and patient was rewarmed. Aortic cross clamp time was 45 minutes. Patient remained hemodynamically stable throughout the case requiring only minimal pressor support and no significant changes were evidenced in the cerebral oximetry monitor or urine. He was transferred intubated to cardiac ICU on low dose epinephrine and milrinone.

Patient was weaned from mechanical ventilation and extubated uneventfully three hours postoperatively. Post-extubation, patient was found to have acute onset left sided hemiplegia with non-fluent aphasia. An emergent CT scan of the head showed multifocal white matter hypodensities with no significant acute changes. All the routine postoperative laboratory findings including electrolytes, ECG and a repeat $\mathrm{CT}$ scan of head were found to be normal. Patient recovered to his baseline neurologic status within 36-48 hours postoperatively. He was eventually discharged to follow standard cardiac rehabilitation after being cleared by speech therapist, physical and occupational therapies.

\section{Discussion}

Cold agglutinin disease (CAD) is a hemolytic process with an incidence of approximately one per million people per year $[4,5]$. CAD is mainly mediated by IgM proteins directed against the " $\mathrm{l}$ "' antigens located on the red blood cells (RBC) surface, which leads to activation of the complement system and allows the C $3 \mathrm{~b}$ molecule to attach to the RBC membrane resulting in agglutination of RBC when exposed to cold temperatures. On rewarming, the IgM molecule gets detached allowing agglutinated erythrocytes to separate from each other while $\mathrm{C} 3 \mathrm{~b}$ remains bound to membrane. A portion of these C3b-coated RBCs is sequestered and ultimately lysed by the Kupffer cells in the liver resulting in extravascular hemolysis [6]. IgM molecules can be polyclonal, typically seen in the post infectious setting (by micro- organisms as Mycoplasma, legionella and Epstein Barr virus) $[4,7]$ or monoclonal. Monoclonal IgM antibodies are classically present in older adults (> 70 years) and it is often associated with an underlying lymphoproliferative disorder in approximately $75 \%$ of the cases $[4,8]$.

The agglutination reaction is composed by two phases: Agglutination with cold, and hemolysis on rewarming. The occurrence of one or the other will vary according to the CAs intrinsic characteristics. The thermal amplitude is defined as the highest temperature at which the CAs react with the antigen. In general, the clinical significance of the CAD depends more on the thermal amplitude than on the titer [5,7-9]. The higher the thermal amplitude results in a more severe presentation of the disease [9]. Cold agglutinins should not be confused with cold agglutinin disease, given that low titer cold autoantibodies (< 1: 40) can be detected in virtually all normal subjects under appropriate conditions and are clinically insignificant [10].

By definition most of the patients with CAD will have anemia, but due to body compensation mechanisms, less than $40 \%$ of patients exhibit symptoms (livedo reticularis, Raynaud disease, acrocyanosis and cutaneous necrosis) which are a result of peripheral agglutination of RBCs during their transit through distal and colder parts of the body [4].

Using strategies to mainly avoid hypothermic exposure, plays the main role in management of CAD $[4,7,11]$. Diverse medical management including corticosteroids, alkylating agents and rituximab have been utilized with different responses [4-8,11]. Ultimately surgical intervention involving splenectomy is not recommended in these patients given that the extravascular hemolysis predominantly takes place in the liver and not the spleen $[7,11]$.

Patients with CAD presenting for surgical procedures might require none or minimal perioperative preparation, however, procedures requiring hypothermia like cardiac surgery and deep hypothermic circulatory arrest carry a significant risk of morbidity [8]. The neuroprotective advantage of hypothermia makes it a favorable strategy in these procedures were it is common to use cold blood cardioplegia below $10^{\circ} \mathrm{C}$, thus exposing the blood to vulnerable temperatures with the potential to cause agglutination and/or hemolysis with subsequent severe anemia, myocardial and other end organ dysfunction [8].

Multiple strategies have been used to prepare and manage these patients exposed this type of procedures. Interventions can be classified according to the portion of the perioperative period that can be used (Table 1).

Thus, CAD poses a unique challenge characterized by multifactorial etiology, myriad of clinical presen- 
Table 1: Perioperative strategies.

\section{Preoperative:}

- Since most of the $\operatorname{lgM}$ is intravascular, some authors have proposed the use of plasma exchange with fresh frozen plasma [4,8-11].

- Determine the specific cold agglutinin thermal-range to guide intraoperative hypothermic limit $[8,9]$.

- Titer levels and hematology consult might be beneficial $[1,8,10]$.

- Patients with very low hemoglobin levels might require transfusions of RBCs and/or erythropoietin to optimize oxygen carrying capacity $[7,8,11]$.

- Maintain normothermia $[1,4,8]$.

\section{Intraoperative:}

- Use of warmed products, including bypass priming fluids, blood products, intravenous fluids and anesthetic gases $[1,4,8-10]$.

- Using body-warming blankets and elevation of operating room temperature $[1,4,8,10]$.

- Intraoperative monitoring of core body temperature to continuously stay above the reactive thermal amplitude of the CA antibodies $[4,10]$.

- Cardioplegia and CBP modifications

- Maintain normothermia or moderate hypothermia above the thermal range $[8,10]$.

- Avoidance of cardioplegia [1,8,12].

- Use warm blood cardioplegia [2,3,8,9].

- Complete coronary and cardiac washout with intermittent cold crystalloid [9,11-14].

- Cold blood cardioplegia in patients with CA and low thermal amplitudes [8].

- If cold agglutinin disease is discovered intraoperatively

- Return patient to normothermia or to moderate hypothermia $[8,13]$.

- Use of warm blood cardioplegia [8,10].

- Switch from blood to cold crystalloid cardioplegia (may provide inferior myocardial protection) $[8,10]$.

\section{Postoperative}

- Keep the patient warm at all times $[4,8]$.

- Be vigilant for symptoms and signs of possible end organ damage [9].

- Transfuse as needed [4,8].

tations, triggers, variable outcomes and diverse management strategies.

We are presenting a unique and interesting case because despite this patient's wide range of CA (-4 to 37 ${ }^{\circ} \mathrm{C}$ ) he was asymptomatic prior to surgery, even with CA antibodies reacting at normal body temperature. It is believed that the cooling of the patient might have led to the activation of the cold-reactive antibody leading to the acute CVA episode. A MRI scan of the brain could have facilitated in confirming the cause of the CVA, however the presence of pacing wires in the patient contraindicated the MRI scan. Learning from such adverse events is critical in our efforts to improve the care we provide.

\section{Conclusion}

Health care, especially hospital-based, is a highly complex integration of multiple providers performing high-risk procedures and administering potentially harmful medications to patients with significant underlying disease. It is not surprising, then, that some patients are harmed by the very health care that is intended to help them. A root cause analysis (RCA) was conducted by the QI division of our Anesthesiology department to identify the key factors that could possibly have contributed this cold-agglutinin relat- ed problem. Initially developed to analyze industrial accidents, RCA is now widely deployed as an error analysis tool in health care. RCA is unique to our residency training and to the best of our knowledge, the Anesthesiology department at UF COM Jacksonville is probably the only Anesthesiology Program in the country to use resident-driven RCAs for scrutiny of adverse events and the teaching of $Q$ l concepts. Based on our RCA recommendations, we were able to implement certain vital changes to help improve patient safety and quality of care.

\section{References}

1. Bratkovic K, Fahy C (2008) Anesthesia for off-pump coronary artery surgery in a patient with cold agglutinin disease. J Cardiothorac Vasc Anesth 22: 449-452.

2. Cho SH, Kim DH, Kwak YT (2014) Normothermic cardiac surgery with warm blood cardioplegia in patient with cold agglutinins. Korean J Thorac Cardiovasc Surg 47: 133-136.

3. Kansaku R, Kuwaki K, Amano A, Inaba H, Tambara K, et al. (2012) Aortic valve replacement to a patient with high titer of cold agglutinin. Ann Thorac Cardiovasc Surg 18: 259261.

4. Swiecicki PL, Hegerova LT, Gertz MA (2013) Cold agglutinin disease. Blood 122: 1114-1121.

5. Berentsen S, Tjønnfjord GE (2012) Diagnosis and treatment of cold agglutinin mediated autoimmune hemolytic anemia. Blood Rev 26: 107-115. 
6. Berentsen S, Sundic T (2015) Red blood cell destruction in autoimmune hemolytic anemia: role of complement and potential new targets for therapy. BioMed Res Int 2015: 363278.

7. Berentsen S, Randen U, Tjønnfjord GE (2015) Cold agglutinin-mediated autoimmune hemolytic anemia. Hematol Oncol Clin North Am 455-471.

8. Barbara DW, Mauermann WJ, Neal JR, Abel MD, Schaff HV, et al. (2013) Cold agglutinins in patients undergoing cardiac surgery requiring cardiopulmonary bypass. J Thorac Cardiovasc Surg 146: 668-680.

9. Agarwal SK, Ghosh PK, Gupta D (1995) Cardiac surgery and cold-reactive proteins. Ann Thorac Surg 60: 11431150.
10. Shah S, Gilliland H, Benson G (2014) Agglutinins and cardiac surgery: A web based survey of cardiac anaesthetic practice; questions raised and possible solutions. Heart Lung Vessels 6: 187-196.

11. Berentsen S (2011) How I manage cold agglutinin disease. Br J Haematol 153: 309-317.

12. Williams AC (1980) Cold agglutinins. Cause for concern? Anaesthesia 35: 887-889.

13. Diaz JH, Cooper ES, Ochsner JL (1984) Cardiac surgery in patients with cold autoimmune diseases. Anesth Analg 63: 349-352.

14. Bracken CA, Gurkowski MA, Naples JJ, Smith H, Steinmann A, et al. (1993) Case 6--1993. Cardiopulmonary bypass in two patients with previously undetected cold agglutinins. J Cardiothorac Vasc Anesth 7: 743-749. 